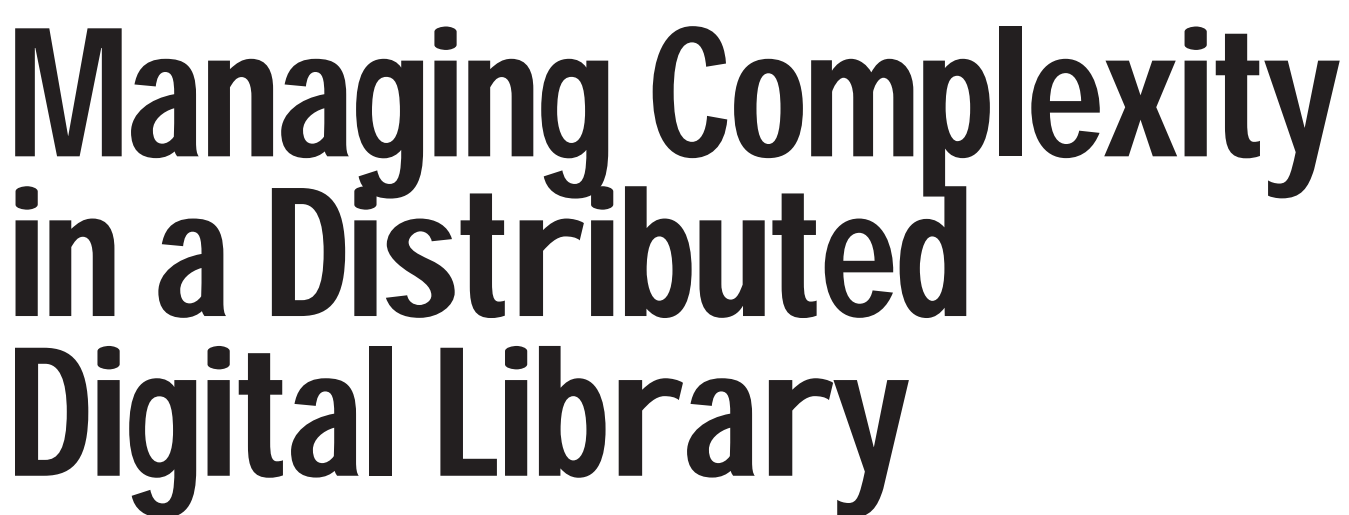

\begin{abstract}
With multiple collections, languages, and media, digital libraries are becoming more difficult to maintain and develop. Researchers at New Zealand's University of Waikato have developed a software architecture that deals with this complexity.
\end{abstract}

I an H. Witten

Rodger J.

$\mathrm{McNab}$

Steve Jones

Mark

Apperley

David

Bainbridge

Sally Jo

Cunningham

University of

Waikato

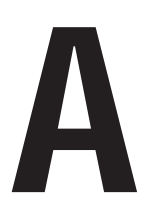

s the capabilities of distributed digital libraries increase, managing organizational and software complexity becomes a key issue. How can collections and indexes be updated without impacting queries currently in progress? W hen users can communicate with the system in different natural languages, how can software cope with the display of various versions of text? With multimedia collections, how can a uniform, extensible software framew ork accommodate different kinds of search engines required to search various media types? H ow can the system handle several user-interface clients for the same collections? $\mathrm{H}$ ow do these clients learn about new collections created in different physical locations?

We have developed a software structure that successfully manages this complexity in our own digital library. It dynamically handles additions of new collections in different locations without requiring a centralized list. Its novel, flexible macro language manages interfaces to many collections in many languages, and the structure allows development of experimental user interfaces. It accommodates radically different search engines within a uniform structure and communication protocol. The architecture has met our goal of minimizing maintenance while allowing our digital library to easily expand its offerings.

\section{THE NEW ZEALAND DIGITAL LIBRARY}

The New Zealand Digital Library (http://www. nzdl.org) currently offers about 20 collections, varying in size from a few documents up to 10 million documents and several gigabytes of text. These collections

- use different browsing and indexing structures;

- are accessibleby computers in different locations;

- support interaction in a variety of languages; and

- contain text, graphics, and audio components.

Some, such as our music collection, require special retrieval engines for nontextual information.
$M$ any collections are updated automatically every few days, but a few-such as the Oxford Text Archive- rarely change. M ost collections are created from electronic source texts, but some- notably the Historic New Zealand N ewspaper Collectionrequirescanning and the use of optical character recognition $(O C R$ ) to capture the source material. Some collections and their contents are w ell structured and lend themselves well to browsing, but many have little or no easily discernable structure.

\section{Multiple languages}

The library's documents are written in many different languages, including English, French, German, Arabic, M aori, Portuguese, and Swahili. M any collections contain several languages, as do some documents. For example, the $\mathrm{H}$ istoric $\mathrm{N}$ ew Zealand N ewspaper Collection, illustrated in Figure 1, is bilingual, with parallel $\mathrm{M}$ aori and English articles.

Thelibrary also provides interfaces to the collections in several languages. For example, the Computer Science Technical Reports collection has English, French, German, and M aori interfaces. The Arabic interface to the Arabic Library, shown in Figure 2, requires storage and search mechanisms that can handle non-A SCII al phabets. Because the code that produces W eb pages is divorced from text strings appearing on them, new languages can be added by merely translating all phrases and menu terms used in the interface.

To accommodate blind (with speech synthesizers) and partially sighted users (with large-font displays), we provide text-only versions of the interface for each language. For this, we follow standard guidelines for Web-page accessibility. ${ }^{1}$

\section{Multiple media}

Some collections contain more than just text. For example, the Local O ral $\mathrm{H}$ istory Collection includes recorded interviews and photographs that illustrate them. Users query summary transcripts. Figure 3 shows results for a query about VE Day. 


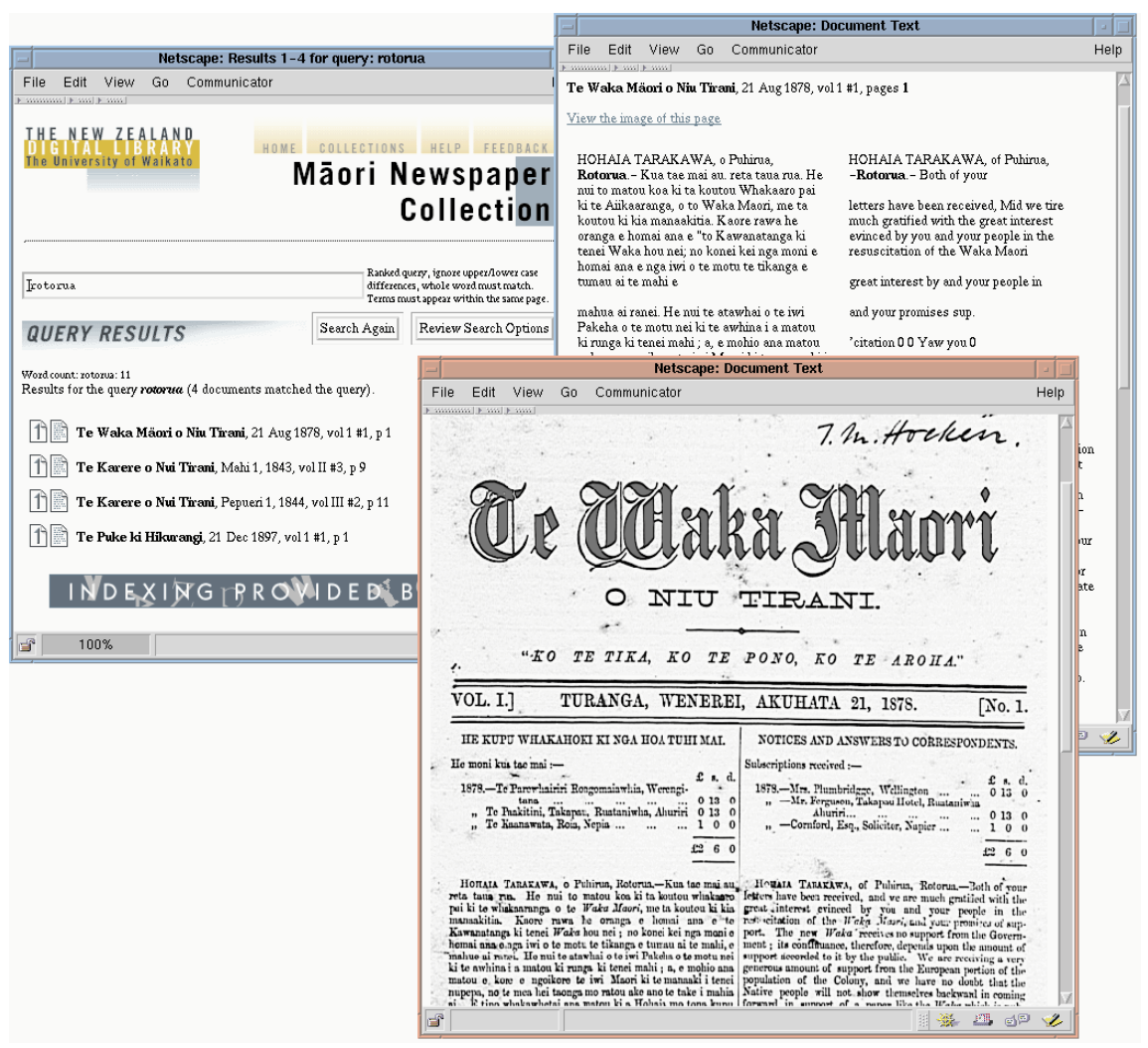

Figure 1. Searching the Historic New Zealand Newspapers Collection. The collection is bilingual; all articles are retrieved in Maori and English.

The M usic Library demonstrates a technically more significant multimedia capability. Users can sing or hum a tune, then upload their audio file to find a match in a tune database. Figure 4 shows the results when a user sings the first eight notes of "A uld Lang Syne." The transcribed input appears at the top left, and titles of similar items, ranked according to how closely they match the query, appear below. A ny of the tunes may be selected for audio replay or visual display, as shown on the right.

The M usic Library database includes nearly 10,000 folk tunes from North America, Ireland, Britain, Germany, and China. Users can search the entire database or limit their searches to individual countries. The M usic Library is a good example of how the digital library's single unifying architecture can encompass radically different search regimes.

\section{SYSTEM DESIGN}

O ur design is based on collections- sets of like documents. ${ }^{2}$ These documents come in a variety of formats: plain ASCII, PostScript, PDF, HTM L, SGM L, and $M$ icrosoft Word for textual documents; Refer, BibTex, and USM arc for bibliographic information; and various formats for graphics and sound.

Collections invariably undergo a building process to make them suitable for search, retrieval, and display. This often involves converting the documents to a different format and identifying subparts that require their own indexes. Among the current collections are indexes for complete documents, individual pages, paragraphs, articles, abstracts, authors, titles, subjects, publication

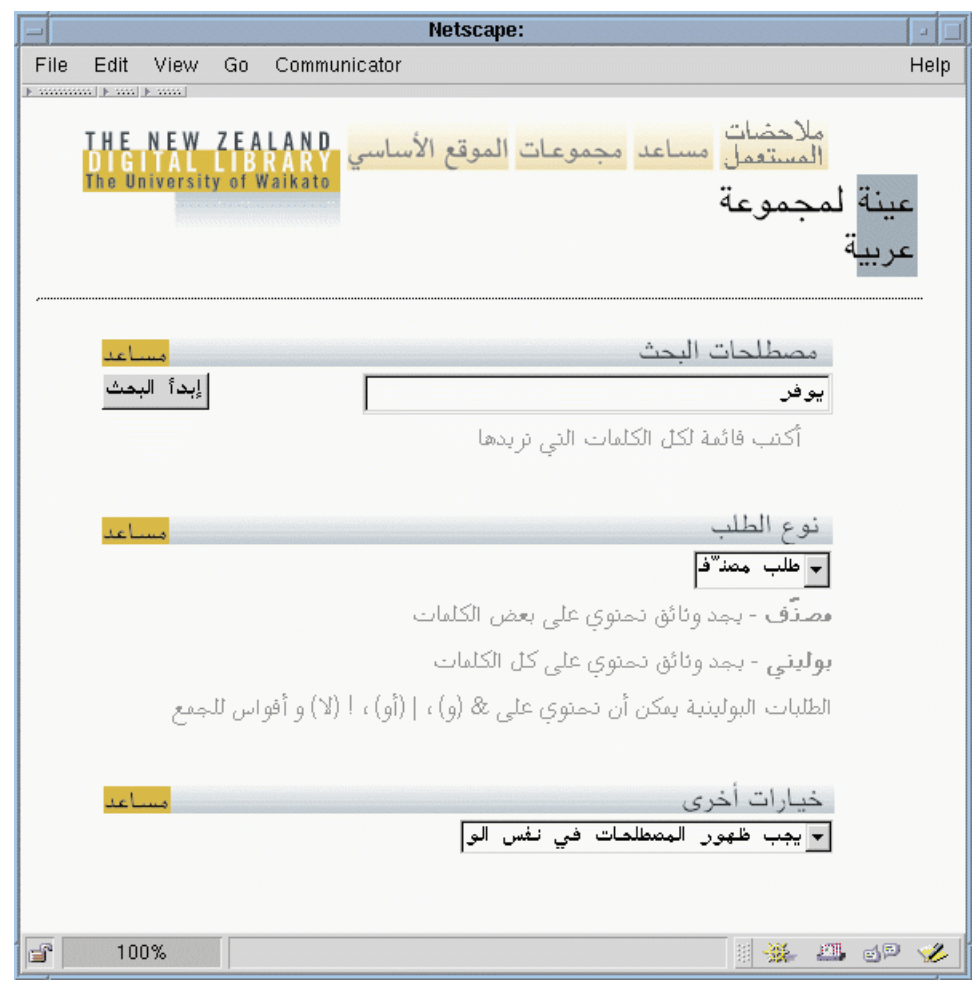

Figure 2. Arabic interface for the Arabic Library.

details, references, and motions in meetings.

Collections are rebuilt when new documents are added to them. To avoid disrupting active users, rebuild- 


\section{Figure 3. Results of a query about VEDay from the Local Oral History Collection. The small gray panel controls audio play- back of a sound bite or relevant section from a participant's interview.}

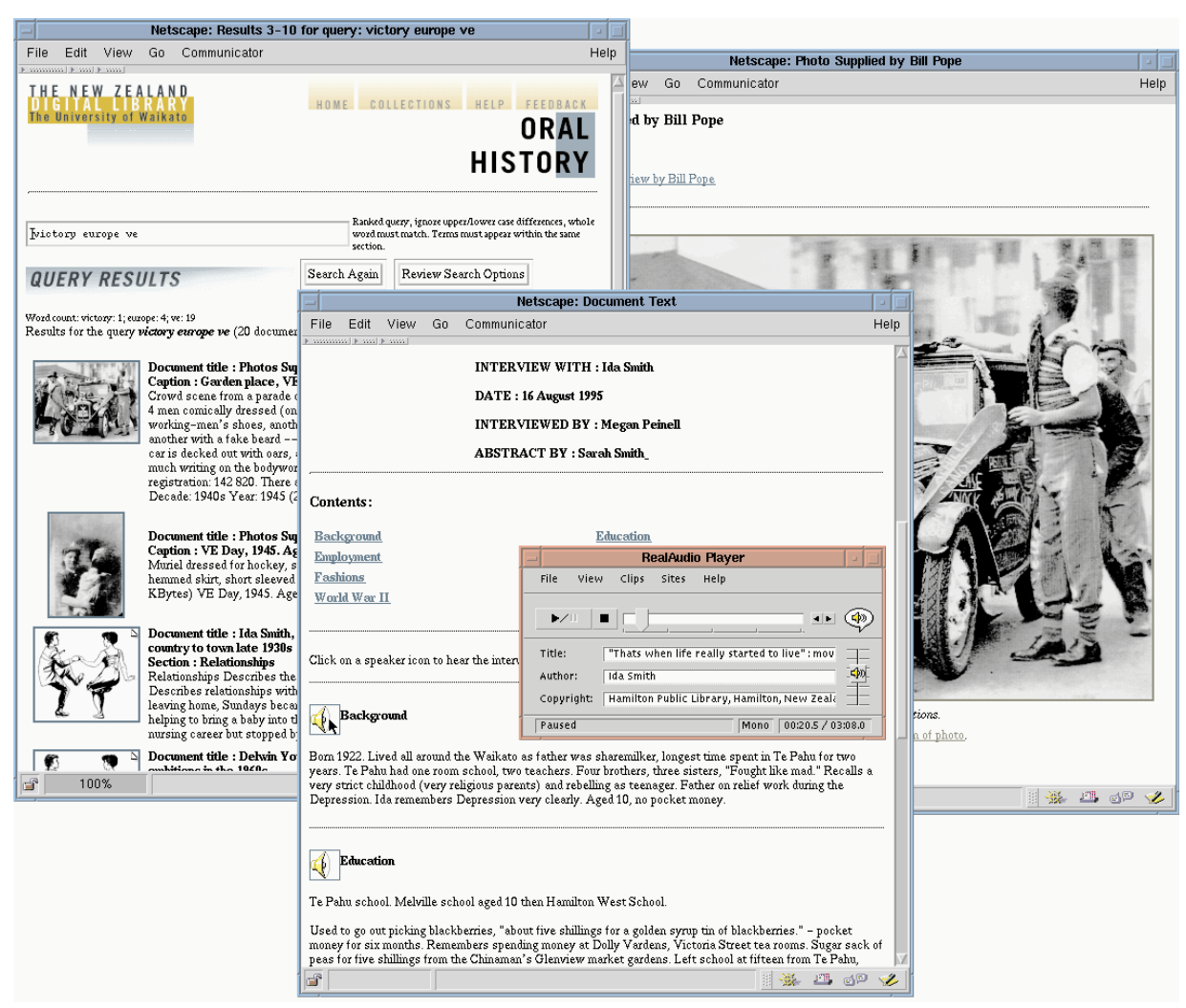

ing takes place in a separate file directory, and when it is complete, the directory is moved in one fell swoop to the live file area. The query engine automatically manages the transition to the new version. Current users experience only a brief delay while the query process reinitializes. R eferences on a query results page, however, may become incorrect after the update occurs. To resolve this, we could simply warn users, but our plan is to instead use persistent document identifiers.

N ormally, several collections operate on the same server machine- by convention in one directory-and each server machine runs its own copy of the collection server software. Collections are active objects, receiving and processing messages, such as search requests and document display requests. Therefore, they can be instructed to look for other collections in their own directory- which avoids a high-maintenance centralized list. Adding a new collection merely involves inserting it into a directory with an already known collection.

W henever a user-interface client starts up (and periodically whileit is running), it messages collections on all machines, requesting information on other collections in their directories. When a collection is added, all interface clients will eventually realize it. This automatic notification process greatly simplifies the process of adding new collections.

\section{Managing complexity}

$\mathrm{M}$ anaging the complexity of multiple collections, multiplelanguages, and multiple interface options presents a significant challenge. For example, document items that have not yet been translated to other Ianguages need to default to English. N on-A SCII languages like $A$ rabic and $C$ hinese need special text positioning and justification. Text-only pages require a structure different from corresponding text-and-graphics pages. Page headers and footers must allow some collections to override them. Directory names and URLS should be referenced symbolically, facilitating the system's configuration to different environments.

For easily maintained, easily augmented, and consistent interfaces to collections, commonality must be abstracted out and represented in just one place. We design Web pages, then, with a model based on structure, content, and reusable items:

- The structure allows different versions of the content. For example, a page might contain some sort of heading, a main information section, and a footer with navigation aids. Theheading in turn might have its own structure, with masthead and navigation aids at the top and an advertisement underneath.

- The content comprises the principal information el ements of the page- text, graphics, sound clips, and video - separated from the page structure in separate resources. $M$ any content items are language-specific, and all resources pertaining to a particular language are collected together for easy maintenance.

- Reusable items include pathnames of certain directories and frequently used icons. Isolating these items renders Web pages more portable. 


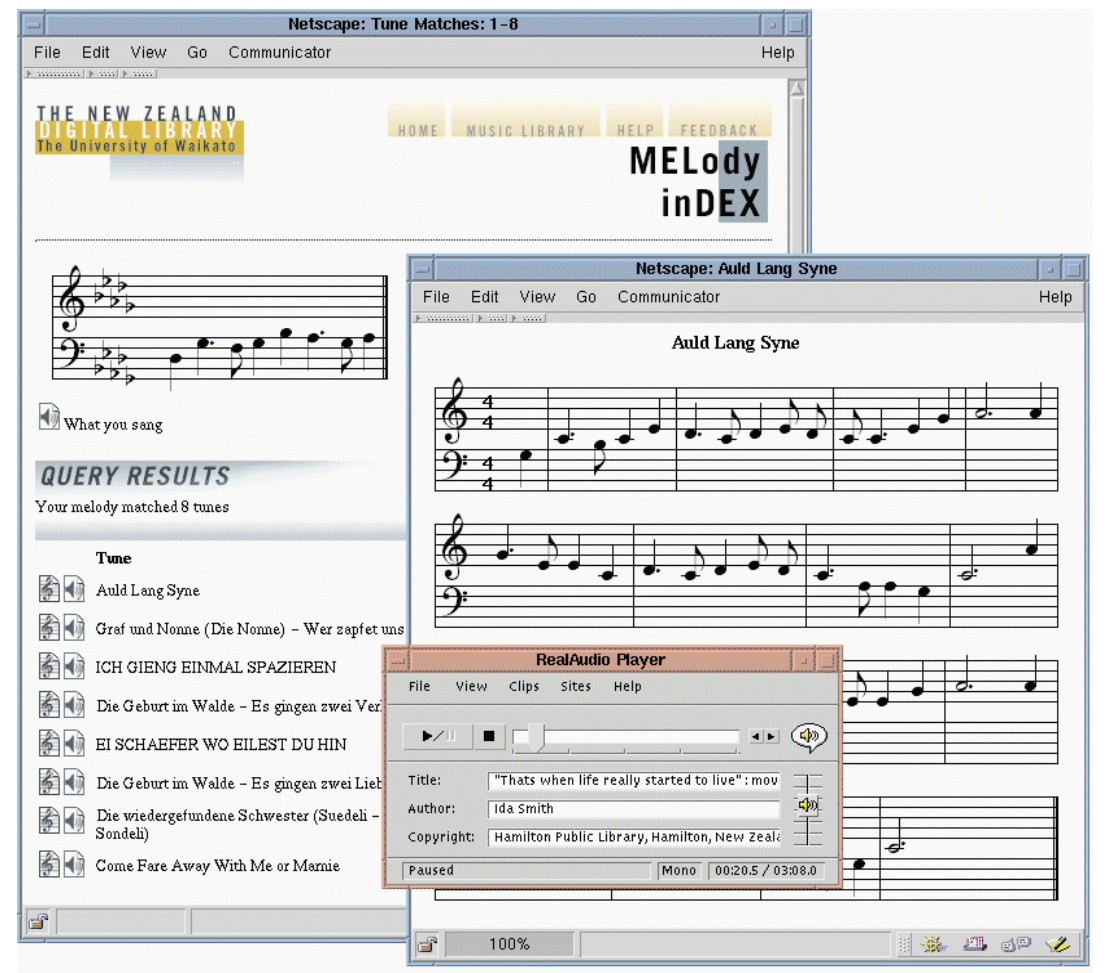

Figure 4. Using the

Music Library to find

"Auld Lang Syne."

We meet these requirements using a flexible, specially designed macro language. While standard macro languages are often used to maintain large collections of Web pages, ${ }^{3}$ such languages only support different page versions through the conditional inclusion of text. This quickly becomes cumbersome as the number of options increases.

O ur macro language, however, allows many variants of the same macro, each with different parameter values. $\mathrm{O}$ ur three basic parameters are collection name, text-and-graphics or text-only format, and language. At expansion time, the macro processor searches the databasefor a variant matching all three parameter values for the generated page's environment. If it does not find an exact match, it uses the closest match based on each parameter's priority. For example, if a user seeks a German text-and-graphics format, but the database contains only English and German text-only versions, the processor calls up the German text-only version.

$M$ acros also support essential maintenance operations. Each week an automatic process scans themacros, locates those that have been flagged as needing translations, and mails the material to appropriatetranslators.

\section{Facilitating searches}

Because collections run on different computers, our system is distributed, but searching is not distributed: Each collection handles its own searches. Cross-collection searching would be relatively easy for collections on the same server, but searching across servers would involve a more difficult distributed search process (which is addressed to an extent by some information retrieval systems ${ }^{4}$ ). 0 ur goal, however, has not been to advance the state of the art in infor- mation retrieval, but to provide a flexible structure that can accommodate the latest advances.

W hile searches in some multimedia collections can be done on textual descriptions of the media objects, different media types generally require different search mechanisms. Presently the system includes two search and retrieval methods-one for text and the other for music. O ur uniform architecture accommodates them and their vastly different document types. This structure is flexible, allowing us to incorporate other retrieval mechanisms in the future-for example, one for accessing an image collection through contentbased queries. $^{5}$

For text, we use M G, a full-text retrieval system that efficiently stores text and its index in compressed form. ${ }^{6}$ M G typically compresses text to about 25 percent of its original sizeand compresses indexes to about 7 percent of the original text's size-making the total storage requirement about one-third of the original text's size.

For music, we use M R, a novel scheme for searching musical melodies. ${ }^{7} \mathrm{M} R$ matches sung (or hummed) input to a database of tunes, with various user options:

- R estricting attention to subsets of the database

- Choosing one of two matching algorithms

- M atching anywhere within a tune or to beginnings only

- M atching using musical intervals or coarser updown-same pitch differential contour

- Ignoring or taking into account note duration, transcribing using fixed tuning, or trying to adapt to overall drift in the user's intonation

- Specifying minimum rest and note lengths

- Specifying music speed 


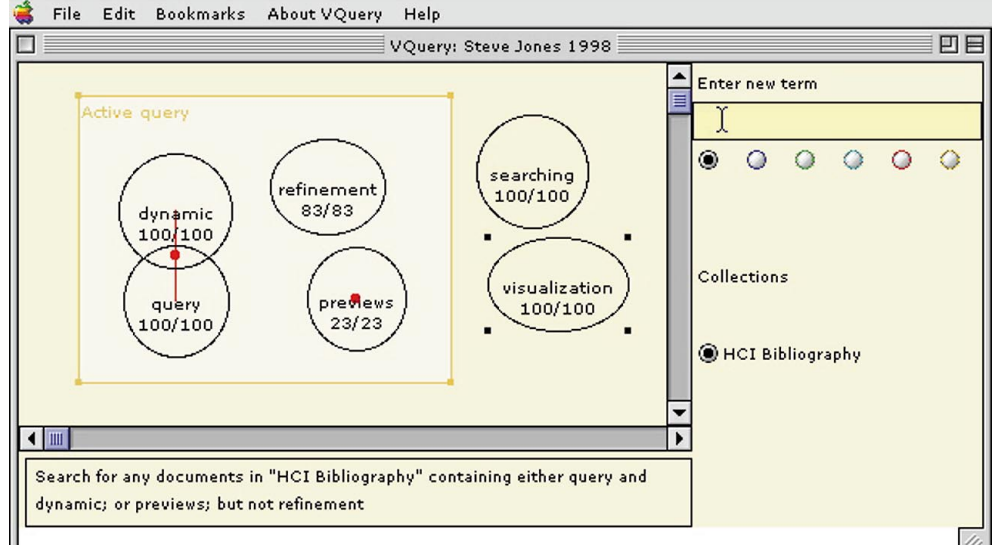

\begin{tabular}{|l|l|}
\hline Sorted by Source & $\hat{\vec{*}}$ \\
\hline
\end{tabular}

Keep selected for later

33 dacuments match the selected query

Query Previeus in Hetworked Information Systems: $t$ Catherine Plaisant, Tom Br. Dynamaps: Dynamic Queries an a Health Statistics A Catherine Plaisant, Uinit. Using Aggregation and Dynamic Queries for Explorin Jade Goldstein, Steven $F$.

(a)

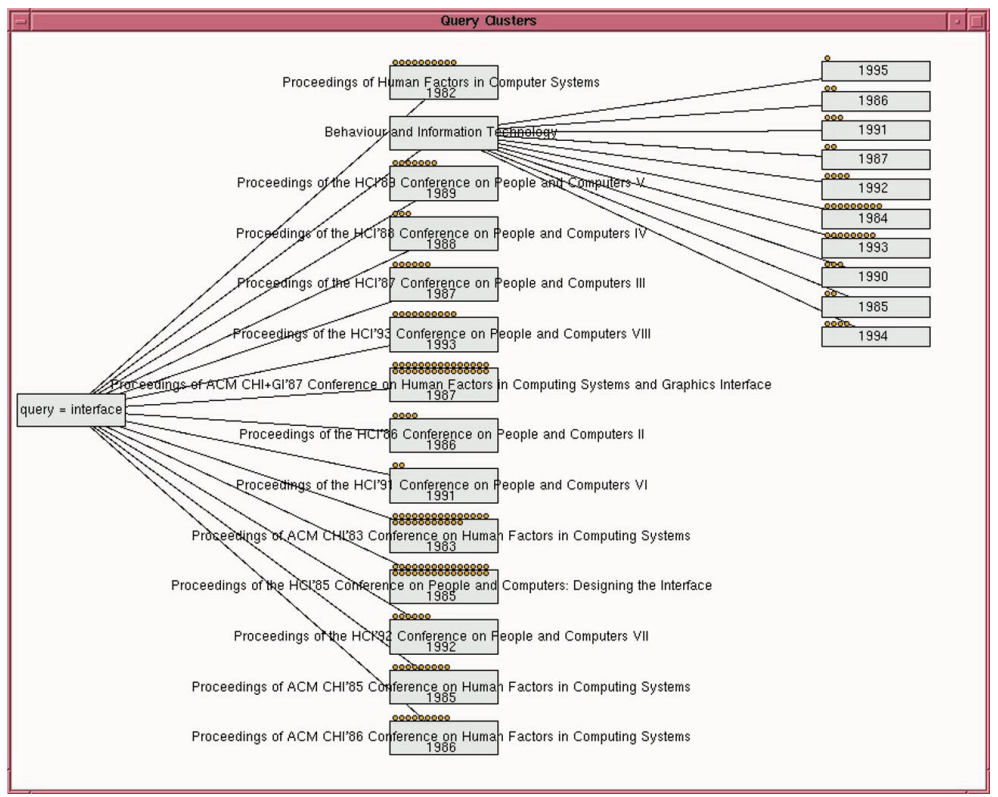

(b)

Figure 5. Two experimental interfaces to the New Zealand Digital Library. (a) $A$ Java applet provides a graphical language for Boolean querying. (b) A Tcl/Tk application visualizes clusters of documents that are the result of a sequence of queries.
$M$ any of these options are needed because users differ in their musical ability and in the accuracy with which they remember tunes.

The process of building a collection is heavily dependent on the mechanisms used to search it because so much of the building involves creating an indexing structure. Consequently, for each collection there is a builder process, which performs the offline activity of creating the index and other supporting structures, and a corresponding collection server process, which manages user interaction.

To accommodate different index structures, often with widely differing needs, the builder and server processes use a flexible, object-oriented program structure. $O$ bject classes with all the basic features needed for building, document retrieval, and searching are defined, but the functionality they provide is selectively overridden by defining subclasses for particular search and retrieval systems. These defining subclasses are themselves finally overridden by collection-specific subclasses that accomplish any task peculiar to a collection.

\section{Improving interfaces}

Internet search engines and online public-access library catalogs commonly provide textual query languages like Z39.58, ${ }^{8}$ but such interfaces present problems for casual users, such as the need to learn the syntax of the language. Using various implementation platforms, we are developing several highly interactive, graphical, and direct manipulation interfaces to the library.

Figure 5a shows one example, a Java applet that provides a graphical language for Boolean querying and updates itself immediately as the query is modified. Figure $5 \mathrm{~b}$ shows another, a $\mathrm{T} \mathrm{cl} / \mathrm{Tk}$ application for visualizing clusters of documents resulting from a sequence of queries. A communication protocol defines how user-interface software communicates with collections through network connections established with collection servers. ${ }^{2}$ These interfaces have been developed without modifying the collection server software and without close code integration between the user interface and the server.

Collection servers recognize three message types:

- R equests for general information, such as details of available collections

- Requests for document information, such as summaries or the full text of documents

- Requests for documents matching specified search criteria

User interface clients do not necessarily operate on any collection's host machine, and our Java implementations are independent of platforms. We have devel oped an application programmer's interface to a software library that contains routines to form and send requests to collections and manage the resulting responses. This makes it easy to integrate new interfaces, without any impact on collection servers. Both the standard Web interface and our experimental interfaces exploit the protocol, and different interfaces can coexist without any difficulty.

Dynamic query interfaces-in which displayed document-result sets change immediately whenever query parameters change - form the focus of our user interface research. Here, operations must be executed quickly on locally cached document information. The protocol allows close control over when information is sent from the collection server to the cache and how much is transferred. For example, in bibliographic collections, items such as author, title, and keywords can be retrieved individually for single documents. Only 
the information that is desired is retrieved over the netw ork. This improves response time, minimizes the size of the local data store, and allows information to be displayed incrementally as it arrives. Users can continue interacting while information is still arriving.

0 ur system successfully manages organizational and software complexity in a large, fully operational, and widely used digital library system. O ur fundamental goal has been to minimize the effort required to keep the system operational and yet continue to expand its offerings. We can now easily build new collections with distinctive features and indexesadding them at any time, on any existing server, while the system is running. We can accommodate new languages by translating phrases and images used in the interface and incorporating them into the collection server software. We can easily incorporate multiple media and initiate nonstandard search engines for them. We can apply new, highly interactive interfaces, implemented on the client sideand using a variety of support technologies. The structure we have developed makes it easy to keep a digital library working and growing.

\section{Acknowledgments}

Wegratefully acknowledge the help of C arl G utwin, who developed one of the experimental user interfaces. $M$ any thanks are also due to Stefan Boddie, Te Taka Keegan, Craig N evill-M anning, and Lloyd Smith, who contributed to this work in various ways.

\section{References}

1. Unified Web Site Accessibility Guidelines, http://www. w3.org/WAI/GL (current Dec. 2, 1998).

2. R.J. M CN ab, I.H. Witten, and S.J. Boddie, "A Distributed Digital Library A rchitecture Incorporating D ifferent Index Styles," Proc. Advances in Digital L ibraries '98, IEEE CS Press, Los Alamitos, Calif., 1998, pp. 36-45.

3. A. Peel, HTM L M acros: Easing the Construction and $M$ aintenance of Web Texts, Tech. R eport 4-96, Univ. of Kent, Canterbury, England, 1996.

4. 0 . de K retser et al., "M ethodologies for Distributed Information Retrieval," Proc. Int'I Conf. Distributed Computing Systems, IEEE CS Press, Los A lamitos, Calif., 1998, pp.66-73.

5. J.R. Smith and S.-F. Chang, "VisualSEEK: A Fully Automated Content-Based Image Q uery System," Proc. ACM M ultimedia Conf., ACM Press, N ew York, 1996, pp.87-98.

6. I.H. Witten, A. M offat, and T.C. Bell, M anaging G igabytes: Compressing and Indexing Documents and I mages, Van N ostrand Reinhold, N ew York, 1994

7. R.J. M cN ab et al., "Toward the Digital M usic Library: Tune Retrieval from Acoustic Input," Proc. Digital Libraries '96, ACM Press, N ew York, 1996, pp. 11-18.

8. NISO, Z39.58: Common Command Language for
O nline Interactive I nformation R etrieval, AN SI/N ISO, Bethesda, M d., 1995.

Ian H. Witten is a professor of computer science at the U niversity of Waikato in $\mathrm{H}$ amilton, $\mathrm{N}$ ew Z ealand. $\mathrm{H}$ e directs the $\mathrm{N}$ ew Z ealand $\mathrm{D}$ igital Library research project. $\mathrm{H}$ is research interests include information retrieval machine learning, text compression, and programming by demonstration. $\mathrm{H}$ e received an $\mathrm{MA}$ in mathematics from Cambridge U niversity, England; an M Sc in computer science from the U niversity of Calgary, Canada; and a PhD in electrical engineering from Essex University, England. $\mathrm{He}$ is a member of the IEEE, and a fellow of the ACM and of the Royal Society of N ew Z ealand.

Rodger J. M cNab is a research programmer for the $\mathrm{N}$ ew Zealand D igital L ibrary. $\mathrm{H}$ is research interests include the architecture of digital libraries, indexing, and digital music libraries. H ereceived a BCM S and an M CM S in computer science from the U niversity of Waikato.

Steve Jones is a lecturer in computer science at the U niversity of Waikato. $\mathrm{H}$ is research interests include digital libraries, World Wide Web navigation, and computer-supported collaborative work, particularly collaborative writing and information retrieval. He received his $\mathrm{PhD}$ in computer science at the U niversity of Stirling, Scotland.

Mark Apperley is a professor of computer science and chair of the Computer Science D epartment at the U niversity of Waikato. $\mathrm{H}$ is research focuses on humancomputer interaction, and his publications address topics ranging from dialogue design notations to interaction devices and computer-supported collaborative work. A pperley is a fellow of the $\mathrm{N}$ ew Z ealand Computer Society and a member of the IEEE Computer Society and of the ACM.

D avid Bainbridge is a lecturer in computer science at the U niversity of Waikato. $\mathrm{H}$ is research interests are computer music and digital libraries. $\mathrm{H}$ e received a BEng in engineering at the University of Edinburgh and $\mathrm{a} \mathrm{PhD}$ in optical music recognition at the U niversity of Canterbury, $\mathrm{N}$ ew Zealand.

Sally Jo C unningham is a senior lecturer at the U niversity of Waikato. $\mathrm{H}$ er research interests include digital libraries, machine learning, and computing education. She earned a PhD in computer science, with minors in A sian history and library science, from L ouisiana State U niversity.

Contact W itten, M cN ab, Jones, A pperley, Bainbridge, and $C$ unningham at $\{$ ihw, rjmcnab, s.jones, m.apperley, d.bainbridge, s.cunningham\}@cs.waikato.ac.nz. 
Dialogue designers

must be both very

careful and very

lucky, or

interaction

problems will remain during the

implementation and

testing stages. 